\title{
Diagnosis de los convenios de cooperación de la Universidad del Zulia*
}

\author{
Montilla, Aída** \\ Prieto, Leticia \\ Arenas, Olga $a^{\star \star \star *}$ \\ Colina, Belinda ${ }^{\star \star \star \star *}$
}

\section{Resumen}

El convenio de cooperación permite desarrollar la relación entre la universidad y el sector externo mediante una negociación. El objetivo es diagnosticar los convenios de cooperación con el sector externo ejecutados por LUZ durante el período 2000-2003, identificando la tipología y caracterizando a los sectores de desempeño (comunicación, económico, educativo, gobierno, industrial, judicial, militar, social, salud) considerados. Es una investigación de carácter exploratorio-descriptivo, mediante revisión documental, siendo la población 266 declaraciones jurídicas, el muestreo fue no probabilístico intencional sobre convenios de cooperación, firmados y renovados durante el periodo de estudio, siendo la muestra, 107 convenios de cooperación, objeto de esta diagnosis. Resultados: el $61,75 \%$ fueron convenios de tipo general, pertenecientes el $55,1 \%$ al sector educativo y $20.6 \%$ al sector Gobierno; el $56.1 \%$ duran 24 meses; el ámbito, fue un 40,2\% al regional y un $37,4 \%$ al internacional; el plazo temporal de $86,9 \%$; el $59,8 \%$ convenios de cooperación académica y el $28 \%$ de apoyo institucional; se realizaron convenios de cooperación principalmente con universidades (35,5\%), seguido de las Alcaldías (14\%). Conclusión, en los convenios de cooperación, expresión jurídica de la relación universidad sector externo, no están definidos de

Recibido: 24-01-06. Aceptado: 15-05-06

* Trabajo financiado por el Consejo de Desarrollo Científico y Humanístico (Condes) de la Universidad del Zulia.

** Profesora Asociada de la Universidad del Zulia, Facultad de Humanidades y Educación. Licenciada en Comunicación Social, Magíster en Planificación y Gerencia de Ciencia y Tecnología. E-mail: amontilla@luz.edu.ve

*** Profesora Titular de la Universidad del Zulia, Facultad de Humanidades y Educación. Psicólogo, Magíster en Gerencia de Empresas. E-mail: Iprieto@luz.edu.ve

**** Profesora Titular, jubilada de la Universidad del Zulia. Facultad Experimental de Ciencias.Economista, Magíster en Economía y Planificación del Desarrollo.

E-mail: ibigilarenas@hotmail.com

***** Profesora Asociada de la Universidad del Zulia, Facultad Experimental de Ciencias. Socióloga, Magister en Gerencia Pública. E-mail: bcolina@luz.edu.ve. 
manera precisos los criterios para establecer el convenio, en el cual la universidad pudiese de manera efectiva acordar el ámbito del desempeño con la contraparte y así obtener los beneficios que declaren en los mismos. Se recomienda, entre otras, diseñar un sistema de criterios explícitos y un sistema de seguimiento para estos convenimientos.

Palabras clave: Convenio, cooperación, sector externo, universidad.

\section{Analysis of Cooperation Agreements at the University of Zulia}

\section{Abstract}

The cooperation agreement allows for developing relationships between the university and the external sector through negotiation. The purpose of this study was to analyze the cooperation agreements made by LUZ with the external sector during the 2000-2003 period, identifying their types and characterizing the performance sectors (communication, economics, education, government, industry, judiciary, military, social, health). The investigation was of an exploratory-descriptive nature, carried out through a review of documents, using a population of 266 juridical declarations; the sampling was intentional and non-probabilistic for cooperation agreements signed and renewed during the period under study, using a sample of 107 cooperation agreements as the object of this analysis. Results: $61.75 \%$ were agreements of a general type with $55.1 \%$ belonging to the educational sector and $20.6 \%$ to the governmental sector; $56.1 \%$ had a 24 -month duration; in terms of geographical scope, $40.2 \%$ were regional and $37.4 \%$ international; $86.9 \%$ were temporary; $59.8 \%$ were agreements of academic cooperation and $28 \%$ for institutional support; cooperation agreements were made principally with universities (35.5\%), followed by Mayor's offices (14\%). In conclusion, in the cooperation agreements which are the juridical expression of the relationship between the university and the external sector, the criteria for establishing an agreement are not precisely defined whereby the university could effectively agree upon the performance field with the counterpart and thereby obtain the benefits declared in the same. Among other recommendations, it is suggested that a system of explicit criteria and a system of follow up be designed for these agreements.

Key words: Agreement, cooperation, external sector, university.

\section{Introducción}

Las universidades públicas enfrentan hoy día una fuerte amenaza que apunta a la esencia del papel y misión que les corresponde tener en la sociedad, para exigir de ésta un desarrollo armónico, equilibrado y con justicia social. El desafío del presente lleva a considerar que toda acción en las universidades estata- les debe tender a desarrollar programas de trabajo que consideren las nuevas tendencias, manteniendo los valores fundamentales que han iluminado su quehacer y que constituyen su aporte a la educación, la ciencia y la cultura.

Esta realidad cambiante ha llevado a las universidades, a implementar diferentes formas de relación con el sector externo de una manera más integradora, incluyendo acciones multidireccionales 
con la finalidad de alcanzar beneficios mutuos, de allí la existencia y proliferación de los convenios. Las universidades, en la necesidad de actualizar sus programas para desarrollar y mantener activa su pertinencia social, el desarrollo y la actualización de su capital humano, han establecido estos convenios que permite una relación universidad sector externo más cónsona con su desarrollo actual (Chiavetta et al; 2002; Santiesteban, 2001; Espinoza, 1999).

Desde esta perspectiva, Espinoza (1999) señala que el sector externo de un país debe beneficiarse de las experiencias y de la investigación universitaria, y las universidades deben analizar la disposición de interactuar con este sector en la generación y transferencia de conocimientos científicos y tecnológicos, generándose entre ambos una relación ganar-ganar.

La idea es acercarse más al ambiente externo y escuchar de este sector lo que necesita, con el fin de aportar docencia, conocimiento e investigación en una alianza estratégica con el sector productivo, transfiriendo conocimientos y tecnologías, con el propósito de aumentar la relación universidad-sector externo a través de convenios que puedan aportar servicios de laboratorio, la formación de equipos multidisciplinarios, fomentar el desarrollo de patentes de invención y el desarrollo de proyectos de mediano y largo plazo con las contrapartes interesadas en la negociación. Las ventajas para la universidad serían poder diseñar programas de formación sensibles a la realidad externa y realizar investigaciones en áreas de desarrollo prioritario para el país, porque con la capacidad universitaria, infraestructura e intelecto y la experiencia de las empresas se pueden hacer cosas muy importantes (Kurte y Retamal, 2002).

Se revela que en las universidades nacionales más importantes del país, la relación universidad-sector externo, basada en el establecimiento de convenio, carece de una metodología para determinar la efectividad de los mismos entre las partes (Espinoza, 1999); ante esta debilidad los resultados de este estudio contribuiría a desarrollar una metodología que considere criterios claros y precisos para el convenimiento, en el cual la universidad pudiese acordar de manera más efectiva el ámbito de desempeño y así, obtener los beneficios que se declaran en los mismos.

En la Universidad del Zulia (LUZ), la relación universidad - sector externo se establece a través de los convenio desde 1967 con el Instituto Agrario Nacional, esta iniciativa crea la necesidad de un ente coordinador, promotor y gestor de esta acción en LUZ, exigencia que es respondida en 1989 a través de la Oficina de Cooperación Institucional; en 1990, se eleva a Dirección de Relaciones Internacionales y desde 1994, hasta el presente, se denomina Dirección de Relaciones Interinstitucionales (DRI), dependencia comisionada ante los entes externos, para establecer la declaración jurídica de los convenios y velar por el cumplimiento de los mismos.

Una de las realidades que confronta la firma de un convenio de cooperación es la interpretación que se hace de la cooperación puesto que existen dos maneras de identificarla: la cooperación conocida como "ayuda" (financiera y técnica) y la asistencial, manejadas a escala inter- 
nacional a partir de las décadas 50 y 60 . El desafío universal es ver cómo se pueden encontrar nuevas formas de cooperación, donde el sistema de educación superior diseñe modelos de negociación y concilie intereses que abran un nuevo modo de trabajar, que beneficie a ambas partes y se logre un valor agregado de carácter social y económico.

El reto que se perfila para el resto del siglo XXI, es resolver los problemas comunes que aún no han encontrado su camino entre la gran mayoría de los políticos y de la ciudadanía, el nuevo sistema de cooperación tiene que nacer, porque hay una necesidad absoluta de generalizar formas de cooperación en interés de todo el mundo. De allí que, las instancias que tengan en su estructura la necesidad de establecer cooperación con sus partes, deberían definir una política coherente y de carácter productivo en el marco de cualquier negociación, para lograr mayor efectividad y, obtener apoyo social y económico entre las partes.

Sobre la base de estos planteamientos y la necesidad de tener un conocimiento de la expresión jurídica de la relación universidad sector externo: convenio cooperación, establecida por LUZ durante el periodo $2000-2003$, se plantea esta investigación, siendo el objetivo general diagnosticar los convenios de cooperación con el sector externo ejecutados en LUZ durante el período 2000-2003. Esto es posible al (a) Identificar los criterios aplicados por LUZ para establecer los convenios de cooperación; (b) Describir los convenios de cooperación ejecutados por LUZ y (c) Caracterizar los convenios de cooperación de acuerdo a los sectores de desempeño.
Los objetivos de esta investigación están orientados hacia la caracterización de los convenios de cooperación y conocer la situación actual en cuanto al proceso de ejecución y, no de una evaluación de la gestión institucional (dependencia ejecutora), con miras al establecimiento de líneas de acción o políticas que en su aplicación ayudarían al cumplimiento de la estrategia general y a alcanzar los factores de éxito de los convenios.

Se hace necesario aclarar que en este estudio, no se analiza el convenio y la cooperación como componentes separados de la relación universidad - sector externo, sino como la expresión jurídica de dicha relación: convenio de cooperación; que se establece a través de mecanismos que garantizan una relativa equidad y reciprocidad en el intercambio o prestación de servicios que establecen las universidades entre si, y entre estas e instituciones y organismos regionales nacionales e internacionales.

\section{Marco referencial de la relación universidad - sector externo}

Desde sus orígenes las universidades han realizado procesos de colaboración e intercambio con instituciones homólogas tanto regional, nacional como internacionalmente; es algo consustancial con sus funciones en materia de docencia, investigación y transferencia de conocimiento e innovación; sin embargo, hasta el presente persisten importantes y notorias debilidades, pues la cultura académica de la universidad está conformada por las representaciones, motivaciones, concepciones e ideas acerca de los objetivos de la 
docencia y la investigación, que condicionan sustancialmente la manera de realizar dichas tareas. Kurte y Retamal (2002) señalan, que entre los principales obstáculos para la relación universidad - sector externo está la fuerte desconexión de parte del mundo académico con la realidad externa, una falta de visión comercial y horizontes de tiempos diferentes. Mientras el mundo industrial busca y necesita resultados rápidos y aplicables a la escala comercial, la universidad trabaja, por lo general, a largo plazo.

La relación de la universidad con el sector externo, se lleva a cabo través de convenio, como expresión jurídica de esta relación, la cual se define según Espinoza (2005), como la declaración de voluntades de dos o más partes de realizar una acción de dar o hacer, se ha establecido hoy en día en una de las manifestaciones más importantes de la actividad universitaria. Ella constituye una forma real de estrechar lazos con la región y el área de influencia del universitario, lo cual permite conocer y sopesar las necesidades del sector externo y de servicios, en cuanto a la aplicación de conocimiento y desarrollo tecnológico, actualización, capacitación y educación continua. Por esta razón, es necesario profundizar teóricamente y prácticamente en el contexto de la relación universidad - sector externo.

Al respecto, desde su enfoque sociotecnológico, Espinoza (1999) señala que es fundamental determinar la necesidad de compartir la capacidad científicotecnológica y humanística para el desarrollo social y económico; y explica la desigualdad que presentan ambos sectores -la universidad y el sector externo- en vir- tud de la posesión y dominio del conocimiento y la tecnología.

Para este enfoque la relación universidad - sector externo, conlleva revisar la concepción de la democratización del conocimiento científico y tecnológico, caracterizándolo como un producto que genera valor agregado en beneficio de la sociedad global; por tal motivo, debe ser accesible al sector externo, con el fin de contribuir a la generación de riquezas para el logro del bienestar social.

Esta desigualdad se puede solventar en parte, con los convenio, expresión jurídica de la relación universidad sector externo, la cual garantiza y facilita la adquisición de conocimientos y el compartir experiencias en igualdad de oportunidades ente las partes (Bossuyt, 2004).

De allí, que en la mayoría de las universidades del mundo la característica primordial es la expresión jurídica convenio con la contraparte, las instituciones y empresas que le permitan un aporte para continuar con su misión, vale decir, generar conocimiento a través de sus investigaciones y de la formación del capital humano que es su más preciado producto, el cual va al mercado que debe abastecer. La principal premisa es aunar esfuerzos para ganar-ganar y obtener beneficios que le permitan mejorar la realidad socioeconómica que atraviesan ambos sectores.

Así como las universidades establecen estas alianzas de cooperación mediante la expresión jurídica convenios, las universidades nacionales, públicas y privadas de Venezuela, como la Universidad del Zulia, establecen entre sí, el convenio de cooperación, teniendo las mismas finalidades con diferentes instancias 
tanto públicas como privadas, de carácter educativo, social y estatal.

\subsection{Clasificación de los convenios}

Para una mejor comprensión de las formas de establecer los convenios y tomando como base lo planteado en los objetivos, en cuanto a la descripción de los convenios de cooperación, se recurre a la clasificación planteada por Dos Santos (2000), el cual señala cuatro aspectos: carácter, ámbito, plazo y materia.

Los convenios de carácter general, son expresiones formales de intención para participar en diversas actividades de relación académica, entre las que se pueden especificar el acceso e intercambio de información sobre los programas académicos de pregrado y postgrado en cada una de las partes contratantes; el intercambio de estudiantes, de lo cual se podrán conceder exoneración de matrícula y otros aranceles relacionados, mientras que sea mutuo; intercambio de profesores, académicos visitantes y personal técnico y profesional que participen en programas de investigación, enseñanza o capacitación; desarrollo de proyectos conjuntos de investigación en disciplinas de interés mutuo; intercambio de información y programas de enseñanza, en especial métodos y técnicas innovadoras en los procesos educativos; intercambio de experiencias y desarrollo de nuevas áreas de investigación y docencia; organización y participación conjunta en seminarios, conferencias y congresos educativos, científicos y técnicos; y el acceso a laboratorios, entre otras facilidades de investigación.

Los convenios de carácter específi$c o$, son aquellos por medio de los cuales, la institución adquiere beneficios, obligaciones o responsabilidades de carácter académico, científico y cultural. En los convenios de este tipo se debe especificar los objetivos concretos que se persiguen, así como, las implicaciones legales y financieras para la Institución.

Dependiendo del ámbito de aplicación, entendiéndose para este estudio como el entorno, el espacio o lugar, donde se desarrolla el convenio, pueden ser universales, regionales o particulares.

Según el plazo, que indica el tiempo por el que va a regir la ejecución del convenio que se establezca, pueden ser temporales y/o permanentes.

La materia para este estudio la conformaría el área específica sobre la cual se establece el convenio, las cuales pueden ser de cooperación académica, prestación de servicios, intercambio cultural, apoyo institucional o de intercambio académico y docente.

\subsection{Referentes teóricos de la cooperación}

Hoy en día existe un capital de conocimientos enorme, a todos los niveles, siendo el gran desafío del sistema de educación superior valorar las fuentes de conocimientos y transformarlos en programas y proyectos aplicables a las necesidades de la humanidad; desde esta perspectiva, la cooperación ha venido a ser un complemento a los esfuerzos de estas sociedades, un apoyo a procesos de cambio en todos los ámbitos de los sectores involucrados. La cooperación, es un espacio de diálogo entre las partes, basado en un mecanismo participativo que permite la inserción de estos actores en los procesos de decisión de la gestión 
del desarrollo académico que se establezca en el convenimiento. La cooperación como una propuesta, debe mantener una gestión flexible, autónoma y descentralizada de las acciones, que permita que las partes asuman su responsabilidad, respetando su autonomía, sus roles y manteniendo la función tanto de la institución demandante como del promotor, con la finalidad de lograr los beneficios mutuos que persigue esta negociación (Bossuyt, 2004).

En la práctica, este proceso debe evolucionar gradualmente, con amplia evaluación de sus experiencias entre las partes que operarán en los distintos niveles académicos del alcance del convenio en cuanto a: programas comunes basados en un conjunto de acciones, promoviendo siempre la cooperación entre las partes y a todos los niveles de intervención, con el fin de lograr la coherencia de las acciones relacionadas con la dimensión de las metas a desarrollar.

Por otra parte, la negociación de cooperación necesita buscar mecanismos para asegurar su continuidad institucional y operativa, para el mejor y mayor uso de sus recursos humanos y financieros, y de esta manera contribuir al auto-financiamiento de sus organizaciones y de sus acciones, reduciendo en lo posible su dependencia de los recursos ajenos.

Ante esta perspectiva, es importante destacar que los nuevos enfoques de la cooperación en la educación superior se caracterizan por un incremento del papel activo de las instituciones, superando enfoques basados en la simple aceptación y participación en esquemas externos de cooperación de oferta. De allí que nuevos enfoques de la cooperación acti- va e integrada institucionalmente, están sustituyendo a los enfoques tradicionales de la cooperación pasiva y espontánea. La cooperación activa requiere el establecimiento de políticas y estrategias gubernamentales e institucionales a fin de que se traduzca en una mejor especificación de los criterios, de los objetivos, la selectividad en la búsqueda de los socios idóneos para garantizar el beneficio mutuo y una tendencia a la diversificación de las modalidades de cooperación y a la cooperación «a la carta» (Bossuyt, 2004).

Teniendo como base los planteamientos anteriores, la cooperación en este estudio se entiende como la actividad que desarrollan diversas Instituciones, a fin de concretar programas, proyectos y convenios de interés común, para la producción del conocimiento y el saber, y la extensión de vínculos existentes entre ellas (Bossuyt, 2004).

Desde el punto de vista integral, la cooperación es el mecanismo de interacción e interrelación de mayor relevancia en el ámbito nacional e internacional, la cual ha logrado desarrollar actividades, que van más allá de las capacidades reales de una institución, a través de las relaciones de intercambio socio-económico y cultural entre naciones e instituciones, para mejorar las condiciones sociales, políticas, económicas y culturales en todas las áreas y campos de la vida humana, con el fin de desarrollar la producción intelectual, esto es: la ciencia, la tecnología y las humanidades, a través de las Instituciones de Educación Superior (Bossuyt, 2004).

Para garantizar la ejecución de esta cooperación se instituye la expresión jurídica a través de convenios de co- 
operación, mecanismo jurídico que garantiza una relativa equidad y reciprocidad en el intercambio o prestación de servicios que se establecen entre las universidades o entre la universidad con instituciones nacionales e internacionales; constituyéndose en un facilitador de las funciones básicas de la acción bidireccional sociedad - universidad.

\section{2.1. Instrumentos Jurídicos de la Cooperación}

Los planteamientos que a continuación se hacen en el marco de la cooperación, tienen como finalidad ilustrar los diferentes niveles en los cuales se materializa la cooperación y sobre los cuales se sustenta esta investigación. La cooperación se lleva a cabo a través de acuerdos o convenios marco interinstitucionales, suscritos por los organismos auspiciadores y ejecutores del mismo.

Los acuerdos o convenios marco interinstitucionales, son instrumentos que establecen las relaciones de cooperación entre los organismos multilaterales, en los cuales se incluyen las áreas de interés mutuo; las modalidades para su ejecución y para el financiamiento de la cooperación y el establecimiento de comisiones mixtas para su seguimiento. A través de estos acuerdos interinstitucionales, quienes lo suscriben asumen también, los compromisos contractuales en relación al ámbito de sus competencias y desarrollo de los mismos.

\section{Los acuerdos complementarios} o convenios específicos, son los instrumentos legales por medio de los cuales se desarrolla un área de cooperación especial y se protocoliza la ejecución conjunta de un programa o proyecto de cooperación técnica y económica. El instru- mento se deriva de los acuerdos marcos interinstitucionales vigentes y surgen de las negociaciones bilaterales en las que se aprueban los proyectos específicos a realizar (S/A, 12. 02.05).

\subsubsection{Modalidades de Coopera-} ción

La cooperación se ejecuta a través de las siguientes acciones:

Técnica: Es la ayuda que se lleva a cabo para apoyar el desarrollo económico y social, mediante la transferencia de técnicos, tecnologías, conocimientos, habilidades o experiencias de una de las partes que tiene un mayor nivel de desarrollo en determinadas áreas que la otra.

Asesoramiento, se otorga o recibe mediante técnicos o profesionales con alto nivel de especialización y calificación para ejecución de programas, proyectos 0 actividades de desarrollo, cuyas acciones están dirigidas a facilitar la solución de problemas científicos y tecnológicos, así como la transferencia e intercambio de conocimientos.

La Capacitación, es una acción educativa de perfeccionamiento y/o adquisición de nuevos conocimientos que tiene por objeto la especialización del capital humano en la institución que así lo acuerde, mediante el traslado hacia el lugar o país donde esté ubicada la contraparte, tendiente a lograr su eficiente participación en el proceso de desarrollo y, es proporcionada mediante cursos y otras modalidades a diferentes niveles; se realiza de manera integral y permanente.

Intercambio de personas. Académicos voluntarios, comprende las acciones de profesionales y/o técnicos que, sin fines de lucro, colaboran en la ejecución de programas, proyectos o actividades de 
desarrollo entre las partes que consideren conveniente su participación.

Donación, es la transferencia a título gratuito, de dinero, bienes o servicios, a favor de la contraparte, así como entidades e instituciones extranjeras y organizaciones no gubernamentales de desarrollo receptoras de cooperación técnica, destinados a complementar la realización de un proyecto de desarrollo.

Financiera no reembolsable, es la cooperación ofrecida por algunas fuentes mediante la asignación de recursos en efectivo, con el objeto de apoyar proyectos $\mathrm{o}$ actividades de desarrollo.

Financiera reembolsable, comprende el financiamiento en efectivo, bajo condiciones de interés y de tiempo favorables, para el desarrollo de actividades económicas especificas.

Cultural, es el apoyo institucionalayuda- destinada a la realización de actividades en algunas áreas culturales, mediante entrega de equipos, donaciones de material, capacitación, o intercambios. Esta cooperación está dirigida a museos, casas de cultura y grupos culturales.

Becas, tiene como objetivo contribuir a la formación de personal técnico, investigadores o funcionarios que puedan desempeñar un papel importante en los países en vías de desarrollo, mediante su formación o capacitación técnica en un país más desarrollado.

Pasantías, consisten en el traslado entre las partes de estudiantes de cualquiera de los niveles educativos, con el fin de conocer experiencias, procedimientos, capacidades, conocimientos o instituciones, en temas específicos.

Eventos académicos: seminarios, cursos y talleres, entre otros, son eventos académicos en los cuales se capacita o entrena a la comunidad de las partes, en un tema de interés para el desarrollo nacional, científico o cultural, funcionamiento de una entidad, o el desempeño profesional o laboral en la entidad para la cual trabajan (S/A, 12 02.05).

\section{Metodología}

La metodología seguida se asumió no como un proceso estático e inflexible, sino cambiante, susceptible de modificación; que se va enriqueciendo en el transcurso de sus etapas. Se realizó un estudio de carácter exploratorio-descriptivo, a través de un diagnóstico de los convenios ejecutado por LUZ (primera fase del proyecto de investigación).

La población de esta investigación se conformó con los diferentes tipos de convenios desde la firma del primero en 1967 hasta el 2003, (doscientos sesenta y seis (266) declaraciones jurídicas), prevaleciendo la modalidad convenios de cooperación, objeto de este diagnóstico. El muestreo fue no probabilística intencional sobre aquellos convenios firmados y renovados durante el período de estudio (2000-2003), lo que hizo una muestra de 107 convenios de diferentes denominaciones. Es necesario aclarar que para la DRI (2001), aún cuando las declaraciones jurídicas tengan diferentes denominaciones, administrativamente se ubican en convenio, por tal motivo, para este estudio se acogerá este criterio y la muestra la conformará ciento siete (107) convenios de cooperación.

Para el diagnóstico se utilizó una ficha de información general de los convenios firmados, renovados o concluidos en 
cada año de estudio, analizando la información contenida en el expediente y en la declaración jurídica de los mismos, se clasificaron los convenios de acuerdo a su denominación, estableciendo los parámetros para estudiar la información generada, basada en el contexto general del convenio, obteniéndose los datos que identifican los criterios, las cláusulas, los principios, los propósitos y objetivos utilizados por las partes.

Se diseñó una ficha técnica para clasificar los convenios de acuerdo al sector de desempeño, así como también, el contexto de cada convenio en cuanto a su denominación e identificación, carácter, ámbito, materia, sector, fechas de inicio, renovación y culminación, duración y plazo.

Hipótesis de trabajo: ¿Existe una metodología que permita definir de manera clara y precisa los criterios para establecer el convenimiento en el cual LUZ pudiese acordar de manera efectiva el ámbito de desempeño con la contraparte, y así obtener los beneficios que se declaren en los mismos?.

\section{Resultados del estudio}

Con la finalidad de dar respuesta al objetivo específico que busca diagnosticar los convenios de cooperación ejecutados por LUZ durante el período de estudio identificando el carácter de los mismos, la denominación y tipo de convenio, materia, sector, duración y plazo, se analizó la distribución de cada uno de los indicadores mencionados.

El diagnóstico mostró que en LUZ, el $95,3 \%$ de los acuerdos firmados, las categorías de declaración, contrato y acuerdo no tuvieron representatividad entre los documentos revisados $(4,60 \%$ en total). Además no se observaron criterios definidos, al menos durante el período de estudio, que justificaran las diferentes denominaciones que aparecen en dichos expedientes; es de hacer notar, que los instrumentos jurídicos encontrados no difieren mucho de los convenio de cooperación, en cuanto a las cláusulas, principios y propósitos.

Para la firma de los convenios se consideran criterios implícitos, basados en valores (prestigio, reconocida trayectoria, solvencia moral, económica y social) que debe reunir la contraparte; sin embargo, en LUZ no se especifican estos criterios para establecer el convenimiento, ni para determinar cuándo y porqué se deben renovar los convenios que culminan el plazo establecido en la declaración jurídica.

LUZ firma acuerdos con 26 universidades que declaran una red de macro universidades entre Venezuela y América Latina, suscrita para la ejecución de planes y acuerdos de mutuo interés, con el propósito de fortalecer la relación entre las partes. Sin embargo, según la información recabada de los documentos, la Universidad del Zulia no ha participado activamente en las actividades que ha efectuado esa Red, observándose que en el expediente no se reporta la productividad o beneficios del mismo.

De los convenios de cooperación ejecutados por LUZ durante el periodo de estudio, 66 son de carácter general; es decir, el $61,75 \%$ son expresiones jurídicas que manifiestan la ejecución de diversas actividades, para tener mayor amplitud en su aplicabilidad. Los convenios de carác- 
ter específico, abordan los objetivos concretos que se persiguen, así como las implicaciones legales y financieras para la Institución, representan un $38 \%$ del total.

En cuanto a la duración de los convenios de cooperación, la categoría de mayor frecuencia fue de 24 meses, presente en el $56,1 \%$ (60 convenios de cooperación), lo que permite inferir, que para esta universidad dos años es tiempo suficiente para esperar logros de los convenimientos establecidos; sin embargo no es una constante puesto que los tiempos de duración varían desde uno hasta 60 meses. El $12,2 \%$ de los convenios duraron un año, mientras que $11,2 \%$ se mantuvieron 36 meses, lo que pudiese inducir a pensar que la Universidad prefiere establecer programas y proyectos a corto plazo, probablemente con la intención de evaluar las actividades realizadas en períodos cortos, permitiéndole obtener una mayor eficacia y de esta manera renovar para ampliar su capacidad de acción.

En cuanto al plazo, entendiéndose como la temporalidad por el que va a regir la ejecución del convenio de cooperación que se establezca, 93 convenios de cooperación, es decir el $86,9 \%$, tiene un plazo temporal, confirmando los resultados anteriores y el resto de los convenios de cooperación (equivalente al 13\%) poseen un plazo permanente, puesto que no determinan el tiempo de culminación, ni su renovación.

Los hallazgos expuestos pudiesen demostrar que la Universidad y el sector externo prefieren establecer una relación temporal, ya que les permitiría, al culminar el convenio cooperación, realizar una evaluación de dicha de dicho convenio, para luego decidir su continuidad; sin embargo, este aspecto no se cubre del todo, puesto que en los expedientes revisados no se observó, en un porcentaje significativo y desde el punto de vista cualitativo, procedimientos que comprueben un sistema de evaluación de estos convenios, ni informes de los resultados de su ejecución.

En lo relativo al ámbito (entorno, espacio o lugar) en el cual se desarrollan los convenios de cooperación, se observa que $40,2 \%$ de los convenios se despliegan en un ámbito regional, seguido del internacional con $37.4 \%$ y el nacional con $22.4 \%$ convenios. Se infiere de esto que la Universidad del Zulia logra una relación efectiva con su entorno inmediato y que las relaciones internacionales son más significativas que las nacionales.

Los 107 convenios están distribuidos en nueve sectores de desempeño: judicial, industrial, militar, comunicación, social, económico, salud, gobierno y educativo. Con el sector que más convenios firmó la Universidad del Zulia, en el período 2000 al 2003 fue el educativo, 59 convenios en total $(55,1 \%)$, cifra que resulta significativamente mayor con respecto al sector gobierno que ocupa el segundo lugar con el $20 \%$. Por su parte, los sectores económicos y salud no varían mucho en sus porcentajes, se firmaron el $4,7 \%$ y $5,6 \%$ de los convenios respectivamente. Asimismo, otros sectores que resultan menos favorecidos en la relación es el sector industrial y judicial con apenas un convenio que representan el $0,9 \%$ cada uno.

Se puede deducir que el sector educativo es para esta universidad la razón social de su desempeño, es su campo de acción natural; sin embargo, no ha descuidado su presencia en los demás sectores, aunque sin la relevancia que 
deberían tener, en especial en los sectores social y salud, que son de prioridad nacional y el de comunicación por ser actualmente de preeminencia global.

Basado en la clasificación que hace Dos Santos (2000), la materia de la que tratan los convenios, se encontró que la cooperación académica alcanzó 59,8\%, de los 107 convenios analizados, seguido de apoyo institucional con $28 \%$, prestación de servicio $6,5 \%$ e intercambio académico y docente $5,6 \%$. Esta información permite concluir que, en este periodo, la cooperación académica lleva a la universidad a concretar programas o proyectos y convenios de interés común con el sector externo, ya sea para la producción del conocimiento y el saber, o la extensión de vínculos existentes entre ambas partes, constituyéndose el convenio en la expresión jurídica de la relación que facilita las funciones básicas de la acción bidireccional sociedad - universidad. En segunda instancia se refiere al apoyo institucional, el cual para este estudio coadyuva al fortalecimiento institucional, brindando sostén a una serie de programas y proyectos establecidos mediante una negociación de ganar-ganar entre las partes.

La Universidad percibe también a las Fundaciones Educativas (6,5\%) como una alternativa para el desarrollo de proyectos y la participación de los profesores e investigadores en las diferentes conferencias o ponencias que se realicen en el país. 21 instituciones poseen muy baja frecuencia de relación con la Universidad, ya que representan cada una el 0,9\%, una modalidad por institución, lo cual abre la posibilidad de que éstas relaciones pretendan solamente cumplir compromisos transitorios.
Un aspecto importante a considerar en este diagnóstico, lo constituye las Instituciones con las cuales firmó acuerdos la Universidad del Zulia durante el período de estudio, son organizaciones sociales o modelos estables y relativamente duraderos de relaciones humanas estructuradas, de allí su impacto sobre el desarrollo de la sociedad. Fueron las universidades las que mayor relevancia alcanzaron, con un $35,5 \%$, representadas en 38 convenios de cooperación, seguido de las alcaldías, con un $14 \%$, es decir, 15 convenios de la muestra estudiada.

A pesar de estos resultados, se evidencia un interés en establecer relaciones con casi todas las instituciones que conforman el sector externo, con actores claves de la región y el país (centros de investigación, productos químicos (sector industrial), Asamblea Nacional, corporación de software, corporación de turismo, fuerzas armadas, Gobernación del Estado, ONG's, Institutos regionales, entre otros).

Dando respuesta al objetivo específico que indaga acerca de las características de los convenios de cooperación de acuerdo a los sectores de desempeño: social, educativo, militar, comunicacional, gobierno, jurídico, salud, económico e industrial, se procedió a cruzar el comportamiento de los indicadores más importantes, con cada uno de estos sectores.

Los resultados mostrados en el Gráfico 1 , muestran que la materia apoyo institucional al relacionarse con el sector educativo alcanza un 10,2\%; sin embargo, con los demás sectores, esta materia presenta un comportamiento diferente, observándose que en los sectores gobierno, comunicación, social, económico y de sa- 


\section{Gráfico 1 \\ Relación entre materia del convenio y sector de desempeño}

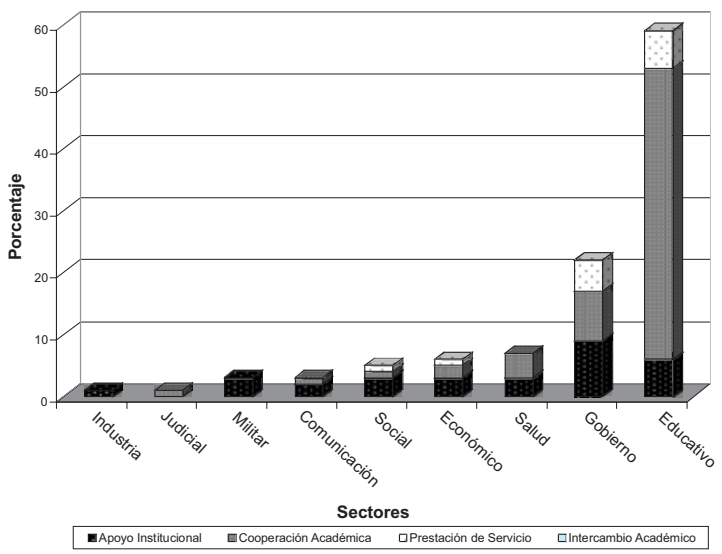

Fuente: elaboración propia.

lud constituye una materia de importancia al momento de firmar acuerdos. Pareciera que en la Universidad del Zulia, dicha materia contribuye al fortalecimiento institucional, brindando soporte a una serie de programas y proyectos establecidos mediante una negociación donde ambas partes ganan y se fortalecen, logrando de esta manera una amplia relación con todos los ámbitos de la sociedad.

En cuanto a la poca relevancia de las materias apoyo institucional e intercambio académico y docente en el sector educativo, posiblemente responda a un campo de acción más centrado en un aspecto del desarrollo, la enseñanza aprendizaje. Con relación a la materia cooperación académica al relacionarse con este sector alcanza $79,6 \%$ lo que permite inferir que esta materia, es quizás, la que mejor se adapta en la relación con las universidades tanto nacionales como internacionales, debido probablemente a que constituye la facilitadora de las funciones básicas de la acción bidireccional sociedad - universidad, garantizando de alguna manera, una relativa equidad y reciprocidad en el intercambio o prestación de servicios que se establecen entre las universidades o entre la Universidad del Zulia con instituciones nacionales e internacionales de este sector. De esto pudiese inferirse que LUZ no tiene criterios definidos para establecer dichos convenimientos con los sectores de desempeño.

En el Gráfico 2, se observa que al relacionar el carácter del convenio de cooperación con el sector de desempeño, el sector educativo posee el máximo porcentaje de convenios de carácter general $(41,12 \%)$, seguido del carácter específico con $14,03 \%$ convenios. Con el sector Gobierno se firmaron $12,16 \%$ acuerdos de carácter específico y $8,41 \%$ de carácter general; el resto de los sectores tiene poca representatividad permitiendo concluir que LUZ, mantiene la intención de relacionarse con los sectores de su entor- 


\section{Gráfico 2}

\section{Carácter del convenio de cooperación según el Sector de desempeño}

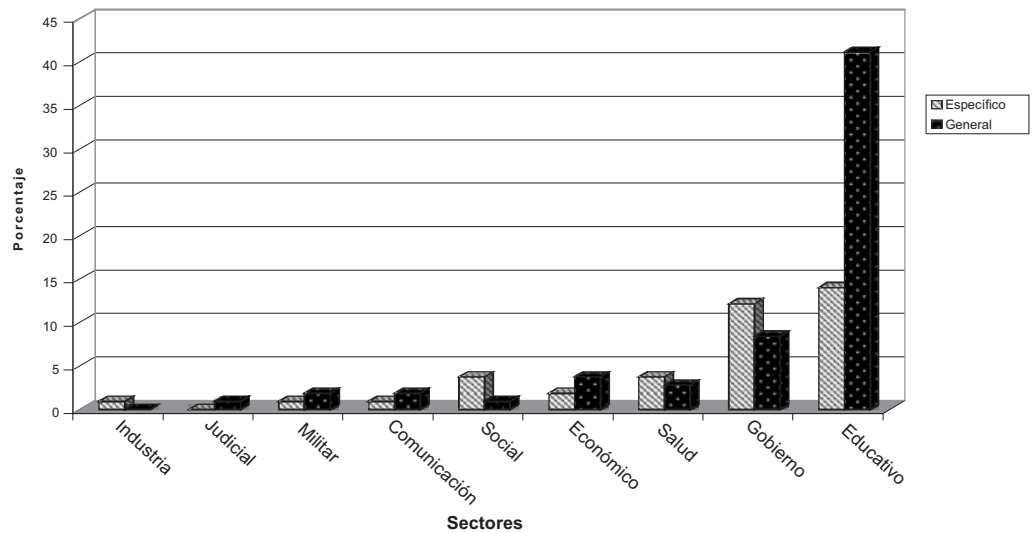

Fuente: elaboración propia.

\section{Gráfico 3 \\ Ámbito del convenio de cooperación según el sector de desempeño}

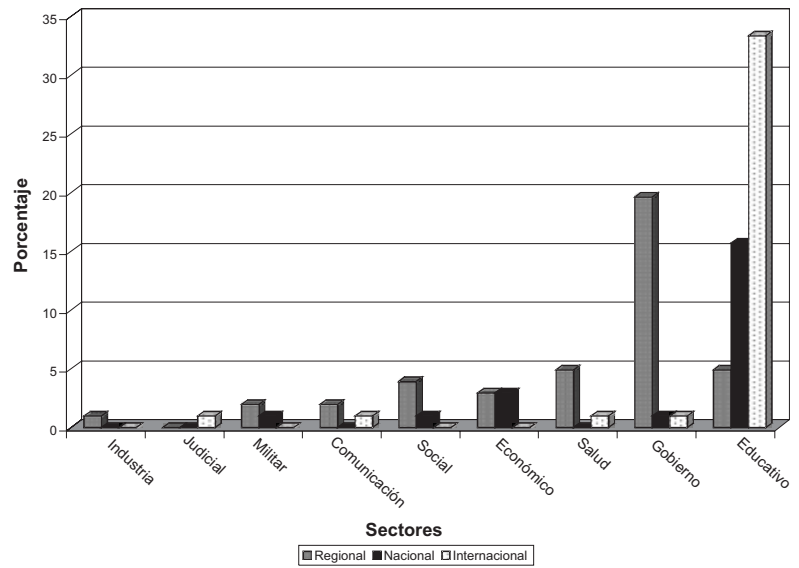

Fuente: elaboración propia.

no, aún cuando no se tiene una relación con mayor capacidad como cabría esperar en una de las universidades de mayor arraigo en la región y en el país.

El Gráfico 3, muestra la relación entre los sectores de desempeño y ámbito del convenio de cooperación, observándose que en el sector educativo se firmaron el $33,34 \%$ de los convenios de ámbito internacional y $15,69 \%$ del ámbito nacional, mientras que en el sector gobierno se encontraron el $19,61 \%$ de los acuerdos 
firmados en el ámbito regional. En el resto de los sectores los convenios firmados en cualquiera de estos ámbitos oscilan dentro de una frecuencia de 1 a 7 , lo que manifiesta que en LUZ estas relaciones no son de mayor trascendencia y que no existe un plan determinado para establecer los convenios de cooperación con las instituciones geográficamente cercanas o lejanas.

Al analizar la relación de los sectores con el plazo o tiempo de duración de las modalidades, se observó que con el sector educativo se firmó el más alto número de convenios en un plazo temporal $(46,15 \%)$, seguido del sector gobierno con $20,19 \%$. En el plazo permanente, también se encontró que es en el sector educativo donde se establecieron mayor número de convenios permanentes (el $10,58 \%$ ), mientras que en el sector gobierno sólo uno fue permanente en su tiempo de duración; en el sector militar dos convenios $(1,92 \%)$ fueron temporales y uno poseía una duración permanente. En los sectores industrial, comunicación, social, económico y de salud todos los convenios firmados fueron temporales, y en el sector judicial el único convenio firmado tuvo una plazo de duración permanente. Se deduce de esto que los acuerdos que estableció LUZ durante el periodo de estudio se enmarcaron en la temporalidad, más que en la definición de acuerdos de mayor trascendencia en el tiempo.

Otro aspecto importante de destacar en el análisis de los documentos jurídicos estudiados lo constituye el hecho de que los datos de las partes declarantes, en los convenios de cooperación son generalmente incompletos con relación a lo estipulado en las cláusulas del documento, ya que se detecta un escaso desarrollo o seguimiento de las actividades que se realizan en el marco de la relación.

En general, la participación institucional de la Universidad del Zulia, como actor de la cooperación, es relativamente reducida, considerando la amplitud del significado de la cooperación, como intercambio y apoyo institucional; esto se evidencia en la carencia de criterios y procedimientos para la elaboración y ejecución de proyectos, la ausencia de ejecutores que se encarguen de hacerle seguimiento a los convenios de cooperación, la falta de un informe que explique los logros obtenidos que permita determinar la renovación y/o culminación de dichos convenios.

La carencia de un financiamiento afín con la ejecución de los proyectos y programas, observados en los convenios, es otra de las principales dificultades para una cooperación más efectiva. La inexistencia de un planteamiento global que determine la dimensión presupuestaria adecuada y el modelo actual de recursos económicos requiere un ajuste cuantitativo y cualitativo, que asegure la unión de los programas y facilite esquemas de financiación, con fondos de la universidad y gubernamentales para el desarrollo de los convenios de cooperación.

\section{Conclusiones}

Se puede concluir que, en el marco de las características de los convenios de cooperación en la Universidad del Zulia, estos reúnen las condiciones como declaraciones jurídicas, sin embargo, carecen de criterios explícitos que permitan que estos sea efectivos. 
No se observaron criterios definidos para la firma, renovación o culminación del contrato que justificara la continuidad de los convenios de cooperación como expresión jurídica de la relación universidad sector externo. Se deduce que lo que orienta estas acciones (renovar y culminar) son los plazos y la duración, más no hay un criterio explícito asumido por LUZ, puesto que no están manifiestos ni definidos, basando la firma de los convenios de cooperación principalmente en valores (prestigio, reconocida trayectoria, solvencia moral, económica y social) implícitos, que debe reunir la contraparte.

La Universidad del Zulia y el sector externo prefieren establecer una relación temporal, probablemente porque les concede la posibilidad de realizar una evaluación de la productividad de la cooperación al culminar el convenio, para luego decidir su continuidad; sin embargo, no se observó resultados de la ejecución de los convenios de cooperación, en relación a informes y productos de los mismos, que permita concluir que la temporalidad fuese un criterio de mayor efectividad.

El carácter o tipo general se acuerda preferentemente en las cláusulas de los convenios de cooperación, siendo sólo declaraciones de principio, objetivos, propósitos que se establecen en el documento, pero que no se materializan o se mencionan porque no existen criterios claros para que estas actividades propuestas se consoliden y puedan obtenerse mejores resultados de los mismos.

En relación con la duración de los convenios de cooperación, la universidad establece dos años como el tiempo máximo ideal; sin embargo, no es una constante puesto que la permanencia varía desde un mes hasta los 60 meses. Se estandarizan los periodos de duración sin tomar en cuenta las particularidades, obviando que cada materia u objeto de convenio es distinta y exige asimismo distintos periodos de tiempo para concretarse; sin embargo, no existen criterios explícitos que permitan determinar que los plazos sean significativos para lograr una efectividad en los convenimientos, al menos en los expedientes diagnosticados. Esto permite concluir que en LUZ, no está definido este criterio como una condición que capitalice el valor agregado de los convenios, para plantearse la posibilidad que a mayor o menor tiempo de duración los mismos serán más efectivos y/o productivos.

En lo relativo al ámbito, el énfasis para LUZ está representado en el ámbito regional, por ser el espacio de mayor influencia, seguido del internacional, sobrepasando al nacional. Esto lleva a inferir que para la universidad el ámbito internacional es de gran trascendencia y, para las instituciones internacionales, LUZ, es un escenario de relevancia para establecer la relación.

Las instituciones con las cuales se establecieron los convenios de cooperación, se clasificaron en ochos sectores de desempeño. El sector que mayor preeminencia logró fue el educativo puesto que las instituciones con las cuales tiene relación son de gran prestigio, tanto regionales como internacionales y a su vez, priva el carácter general del convenimiento.

En lo relativo a la gama de Instituciones, con las cuales se relaciona la universidad, se determinó que no existe un criterio definido de las condiciones que debe tener una institución para estable- 
cer un convenimiento que pueda dar un valor agregado a esta relación, a excepción de que éstas muestren interés en establecer relaciones con LUZ.

En síntesis, la Universidad del Zulia, a través de los convenios firmados, renovados y finalizados durante el período de estudio, no presenta criterios definidos para establecer dichos convenios, perdiéndose quizás, la oportunidad que representan estas relaciones con el entorno de hacerse sentir e influir en el desarrollo de la región, del país y en el ámbito internacional y a su vez, lograr un valor agregado para el desarrollo interno de la misma, con la participación de instituciones de gran importancia con las cuales, LUZ mantiene dichos convenios.

La Universidad no presenta una estrategia para capitalizar los recurso externos y obtener beneficios compartidos de mutuo desempeño, se observó que los convenimientos se firman sólo para unas acciones generales, pero no con base a un plan estratégico de desarrollo donde la realidad científica, social y económica demande el establecimiento de vínculos estrechos entre la universidad como generadora de conocimientos y los sectores productivos (sectores de desempeño) que los transforman en tecnología.

Se observó que aunque la universidad orienta su mayor esfuerzo en el sector educativo, es asumido de una manera tradicional y sin un criterio amplio, aún cuando es un aspecto importante para el desarrollo tanto económico como social de un país.

\section{Recomendaciones}

Los resultados de la investigación realizada, permiten sugerir una serie de recomendaciones, a ser implementadas a corto plazo, para que los convenios de cooperación se consolide como una estrategia viable y productiva para la Universidad del Zulia:

1. Diseñar un sistema de criterios explícitos para establecer los convenios de cooperación.

2. Definir las estrategias de seguimiento para el cumplimiento de los convenios de cooperación.

3. Diseñar una base de datos actualizada (memoria tecnológica) de la relación universidad - sector externo de los convenios de cooperación y el fortalecimiento de un sistema de cooperación interno que involucre en forma activa a todas las unidades académicas internas y regionales, integradas de tal manera, que permita el intercambio de información oportuna para establecer estrategias de coordinación efectivas, a efecto de utilizar en forma oportuna los recursos de la cooperación externa.

4. Conformación de equipos multidisciplinarios e interinstitucionales, que permitan no sólo la presencia de docentes y empresarios, sino de estudiantes y egresados profesionales, presentes en las diferentes regiones, para que la ejecución de los convenios de cooperación sea más efectiva. 


\section{Referencias Bibliográficas}

Bossuyt, Jean. (2004). "Todos los países de las tierra estamos en desarroIlo".Ver enhttp://www.radiolibertad.org/rl/ rep.php?var= nov 2004

Chiavetta, David.; Christlieb, I.C.; Martínez F., M.; Seco M., Rosa M. (2002). Relación universidad sector productivo en el marco del desarrollo sustentable. Ver en http://www. iacd.oas. org/LaEduca132/martinez/martinez133.htm. Febrero 02.

Dos Santos, Daniel (2000). Metodología de medición y mejoramiento de la productividad empresarial. Indicadores de gestión de procesos. Ver en http://www.unap.cl/unico/docs/manual_indica.htx_30-3-03.

DRI (2001). Dirección de Relaciones Interinstitucionales. Plan de Desarrollo Estratégico. Universidad del Zulia. Maracaibo. Venezuela.

Espinoza, Rafael (1999). Naturaleza y Alcance Universidad- Sector Productivo. Venezuela, Editorial de la Universidad del Zulia (EDILUZ).
Espinoza, Rafael (2005). Sugerencia y Recomendaciones. Presentación del proyecto "convenios de cooperación universidad sector externo. Evaluación del impacto interno". En la Línea de investigación del Doctorado en Ciencias Humanas, Facultad de Humanidades y Educación. Universidad el Zulia, Maracaibo Venezuela

Kurte, Patricio y Retamal, Rosario (2002). ¿Por qué las industrias no recurren a las universidades para resolver sus problemas? Ver en http://www. puc.cl /noticias /ficha/pub524.html. 23.03.05

Santiesteban, LI. (2001). Relación Universitaria. Seminario "Relación de la Universidad al sector productivo. El papel de las organizaciones Universidad -Empresa en íberoamérica". Asociación colombiana de Universidades (ASCUM). La asociación Universitaria Iberoamericana de Postgrado (AUIP) y la Universidad de salamanca. Cartagena de Indias. Colombia.

S/A.http://www.concytec.gob.pe/cooperacion/COOPERACI\% D3N\% 20INTER NACIONAL-WEB-SITE.doc Consultado: 1202.05 . 